\title{
Analysis of Relevant Research Progress of Intelligent Sports Industry
}

\author{
ZHAO Yaping ${ }^{1, *}$, CAI Jie ${ }^{2}$ \\ ${ }^{1}$ Library Editorial, Shandong Sport University 250103 Shandong, China \\ ${ }^{2}$ School of physical education, Shandong University 250102 Shandong, China
}

\begin{abstract}
Intelligent sports industry is the organic combination of intelligent industry and sport industry, and it is industrialization performance of intelligent sport. Through literature analysis method and logical interpretation method, four aspects are sorted out into intelligent industry, sport industry, intelligent sport industry and intelligent sports industry, so as to clarify the current academic history and research dynamics of intelligent sport industry. Found: the overall trend of intelligent industry is analyzed, and the corresponding development path and development mode; different industries develop intelligent industry in different directions, and the lack of research, the specific industry, especially the sportindustry.
\end{abstract}

\section{REVIEW OF INTELLIGENT INDUSTRY}

\subsection{Intelligent Industry concepts}

The concept definition of "intelligent industry" is divided into two kinds: relatively narrow definition and relatively broad definition: one is relatively narrow intelligent industry, intelligent industry with creative industry, that intelligent industry simply solely on human wisdom for research and development and creation; the other is related broad intelligent industry, that the power source of intelligent industry is not only limited to the wisdom of science and technology, and that the intelligent industry is intelligence intensive and technology intensive industry [1][2]. This study mainly adopts the second broad definition of intelligent industry.

\subsection{Intelligent Industry characteristics}

Intelligent industry has two characteristics of high science and technology and high industrial integration (Sun He, 2I017). The highly technical refers to the basic and fundamental support of the intelligent industry is the modern information technology, including the Internet, cloud computing, big data, the Internet of Things, etc. The high industrial integration refers to the intelligent industry breaking through a single industry, is a new industry of multi-industry crossover integration, including the emerging information, knowledge, creativity, and the new forms of new technology for the intelligent transformation of traditional industries.

\subsection{Development status of intelligent industry at home and abroad}

The development of the American intelligent industry began with manufacturing informatization in the $1990 \mathrm{~s}$. In June 2011, the US government established a priority action plan in four aspects of the modeling and simulation platform construction, data collection and management standards construction, the optimization of supply chain performance construction and the construction of intelligent manufacturing education, which further clarified the development direction of the intelligent industry. In December 2010, the Ministry of Economic and Technology of the German Federal Government developed a new information strategy,Digital Germany 2015, proposing new economic growth and employment opportunities through digitalization. In July 2009, Japan IT Strategy Department developed the medium-and long-term information technology development strategy "i-Japan", Through the integration of information and communication technology and industry, it fundamentally improved production efficiency, improved the added value of products, develops new markets, and made Japan's economy maintain the leading position in the global world.

In October 2010, the Decision on Accelerating the Cultivation and Development of Strategic Emerging Industries issued by the State Council of China pointed out the organization and implementation of major application demonstration projects such as "Health" for all "and" intelligent manufacturing ", and accelerated the cultivation of strategic emerging industries into leading and pillar industries. Then "about accelerating the depth of informatization and industrialization fusion opinions" on "Internet" + "guidance" "Internet +" artificial

\footnotetext{
$\overline{{ }^{*} \text { Corresponding author: 375283040@qq.com }}$
} 
intelligence three years implementation plan ", the national new generation of artificial intelligence development plan and other policy documents, combined with a series of intelligent city, the Internet of things and other related policies, vigorously develop the wisdom industry from the policy.

\subsection{Intelligent Industry}

The rise of the scholarly industry has had a profound impact on the traditional manufacturing and service industries. China has the world's largest and fastest growing intelligent industrial market in the world, and will show the following development trends and gradually develop in depth. First, the gradual specialization of products: in the future, the vertical industry of intelligent industry will continue to deepen, refine, make industry boundaries and division of labor clearer, and the further clarity of the business field of intelligent enterprises will also make intelligent products gradually become professional. Second, the chain will be gradually extended: the industrial chain will be expanded from intelligent hardware and other hot fields to intelligent service, intelligent city, intelligent tourism, intelligent manufacturing and intelligent life, and will extend from the upstream research and development, production and manufacturing to the final consumers and users downstream, and the industrial chain will be further improved. Third, the market is gradually structured: the industryl line and product direction are constantly clear. The competitive enterprises will stand out, to form a certain distribution and pattern.

To sum up, research of intelligent industry mainly focuses on the conceptual analysis, feature analysis, technology research and development, which provide a theoretical basis for the research and development of China's intelligent industry. However, there are the following problems: the overall trend of the intelligent industry is analyzed, but the specific development direction of the intelligent industry is lacking, different industries have different directions, and the analysis of the intelligent industry.

\section{RELEVANT REVIEW OF THE SPORTS INDUSTRY}

\subsection{Sports Industry concepts}

Some Chinese and foreign researchers have defined the concept of the sports industry in the "broad caliber" way. Although their expressions are altered, there are similarities in the generalization characteristics of industrial extension. "The broad sense of the extension of the sports industry" refers to the collection of operation, production and other departments connected with sport. That is to say, sports industry refers to the collection of ontology industry, related industry and sports industry, which reflects the collection of sports services and sports management activities, related industries refers to sports, including sports media, sports advertising, sports clothing, sports venues, etc.; sports industry refers to the sports department to increase income organization.

\subsection{Sport industry divisions}

According to the definition of the extension and connotation of the sports industry, according to the actual needs of the society and the departments of supplying sports services, follow the principles of convenient economic statistics and economic accounting and relevant international practices, the National Sports Industry Statistical Classification divides the sports industry into 11 categories, 37 middle categories and 52 small categories[3]. Among the 11 categories are: sports management activities, sports competition performance activities, sports, fitness and leisure activities, sports venue services, sports intermediary services, sports training and education, sports media and information services, other sports related services, sports goods and related products manufacturing, sports goods and related products sales / trade agent and rental sports site facilities construction.

\subsection{Development status of the sport industry}

\subsubsection{Sports Industry Policy Environment}

In recent years, the national policy has focused on exploring the huge space of the sports industry, use the development of the sports industry to stimulate domestic demand, promote consumption, increase employment, vigorously encourage the integration of the sports industry with cultural creativity and design service industry, and guide social capital to the sports industry with great development potential.

\subsubsection{Overall scale of the sports industry grows year by year}

In 2014, the total output of national sport and related industries was RMB 1357.471 billion, and the added value was RMB 404.098 billion, accounting for $0.64 \%$ of GDP in the current year. In 2015, the total output of sport and related industries was 1.7 trillion yuan, realizing an added value of 549.4 billion yuan, accounting for $0.8 \%$ of the current year. In 2016, the total output of national sport and related industries was 1.9 trillion yuan, with an added value of 647.5 billion yuan, accounting for GDP growth to $0.9 \%$. The year 1.36 trillion in 2014 grew to 1.9 trillion in 2016, with an average annual increase of $18.2 \%$, exceeding the actual average annual growth rate of around $16 \%$ from 2006 to 2013. The overall scale of the sport industry has increased year by year, but GDP accounts for a relatively low proportion, and there is still much room for growth compared with the average data of $3.5 \%$ of developed countries. It can be seen that the development prospect of the sport industry is very broad. 


\subsection{Sports Industry Development Trend}

The development of sports industry mainly presents four trends: First, the internal logic of the development of sports industry is gradually clear, and the capital logic and media logic of the development of sports industry and the essential internal logic of supporting the development of sport industry will be more and more clear[4]. Second, the integration trend of sport and related industries is constantly increasing. As a part of great health and leisure, sport is an industry with strong integration characteristics. The integrated development of sport and other industries has great potential. Three is the sports new demand, new formats, new model will continue to emerge, in the global new generation of information technology revolution and new industrial revolution integration under the docking background, market-oriented, with technology, application and mode innovation as the core and mutual integration of new economic forms will develop, sports industry as the recent rapid development of emerging industry categories, backwardness advantage is also more obvious. Fourth, science and technology and finance will become the wings of the sport industry to take off. On the basis of following the objective development law, the sports industry cannot be separated from the solid support of science and technology and finance.

To sum up, research of sport industry mainly focuses on the development status and trend of sport industry, which is relatively mature. However, there are several problems in the research of sports industry: current development mode of sports industry, but lack of more specific development measures, and need to construct an operational development mode for specific development trend; the new trend of sports and related industries has emerged, but few new trends for cross-border integration and integration development, and further research on emerging integration of sports and technology, sports and health.

\section{RELATED REVIEW OF INTELLIGENT SPORT}

\subsection{Intelligent Sports concepts}

"Intelligent sport" represents a new field of the development of sports information in the Internet era. But "intelligent sports" is currently without a recognized unified definition, Combining foreign research and our reality, "intelligent sports" can be understood as processing and analyzing the Internet of Things, the Internet, by applying "intelligent technologies" to big data and cloud computing, Intelligent data and other "intelligent technologies", including cloud computing, in various sports fields including competitive sports activities, sports activities, mass sports activities, sports industry, sports culture and sports facilities, to process and analyze mass sensing information, Make intelligent response and intelligent decision-making support to the needs of sports in various fields, including competitive sports activities, mass sports activities, sports industry, sports culture, sports facilities and so on. Some scholars have also pointed out that intelligent sports should be a relatively advanced ecosystem based on big data, cloud computing and Internet of Things technology, integrating competitive sports, national fitness, sports industry, and integrating education, medical care, tourism, culture and other 'sports + ' resources as the basic architecture [5].

\subsection{Intelligent Sports Development Environment}

In his report to the 19th National Congress of the CPC, General Secretary Xi Jinping twice mentioned the content of the intelligent society, and clearly proposed: to promote the deep integration of the Internet, big data, artificial intelligence and the real economy, and to provide strong support for the cyber power, digital China and the intelligent society with technological innovation. The Fifth Plenary Session of the 18th CPC Central Committee put forward the five development concepts of "innovation, coordination, green, openness and sharing", implement the "Internet + " action plan, develop the sharing economy, and implement the national big data strategy. This reflects China's will and determination to actively build an intelligent society and an intelligent country. Intelligent sport, as one of the important contents, will inevitably be fast.

In the context of the rise of national fitness into a national strategy, the people's sport demand is more diversified, and the sport consumption market is bigger. The demand for fitness and leisure, competition, performance, venue services, equipment manufacturing, service trade, sport tourism and sport rehabilitation will grow rapidly. The new round of the scientific and technological revolution with information technology as the core has had a profound impact on all fields of the sport industry. Big data, cloud computing, Internet of Things, e-commerce, and Internet finance have formed a coercive mechanism for the development, transformation and upgrading of traditional sport, and new ideas, new ideas, new ideas and new technologies are urgently needed. intelligent sports construction is the inevitable choice of sports actively adapt to the new normal, into the "Internet + " action plan, the mobile Internet as a leading innovation driven development, promote online and offline deep integration, fully mining, integrate, configuration of all tangible and intangible resources, constantly produce new industrial formats, new business model, new technical means, create diversified services and products, lead the new pattern of sports development, accelerate the transformation and upgrading of sports, promote sports work to a new level.

To sum up, the research of intelligent sport has just started. The research mainly focuses on the concept and preliminary practice, and there is still a lot of room for the research of intelligent sport. There are also the following problems in the research of intelligent sports: the current research studies the future development trend of intelligent sports, but it lacks the analysis of the specific operation mode, business mode and business mode of intelligent sports products or services, and also 
needs to industrialization intelligent sports as an emerging industry type.Lacks research on intelligent sport development content, development carrier and other specific development directions of integration platform.

\section{RELEVANT REVIEW OF INTELLIGENT SPORTS INDUSTRY}

\subsection{The Intelligent Sports Industry Concept}

Intelligent sports industry is the application of a new generation of information technology, sports technology and high intelligence technology, including competitive sports, leisure sports, sports venues and facilities to provide intelligent products and services, help the transformation and upgrading of modern sports industry, is the science and technology industry, cultural industry, traditional sports industry, media industry, biomedical industry, pharmaceutical industry and tourism industry. Can drive from upstream technology research and development (intelligent chip, virtual reality, action capture, holographic display, the Internet, the Internet of Things, biotechnology, genetic testing technology, wearable devices, etc.), to related sports competition, sports fitness, entertainment, sports tourism services, etc., as well as downstream hardware equipment manufacturing, software content design and production, cloud information big data management and other fields of multi-level industrial chain collaborative development.

The concept of intelligent sport industry is based on the concept of intelligent industry integrated into intelligent sport factors. From the perspective of industrial structure, intelligent sports industry is a subindustry category affiliated to the intelligent industry, which belongs to the category of intelligent industry and is highly related to the intelligent industry. Correspondingly, the characteristics and connotation of the concept of intelligent sport industry are inseparable from the concept of intelligent industry.

Scholars define the concept of intelligent industry is mainly as follows: intelligent industry refers to digital, networking, informatization, automation, intelligent degree of industry, is intelligence intensive industry, technology intensive industry, intelligent industry, including research and development design, intelligent production and manufacturing, intelligent management, marketing, its related concepts cover the functional manufacturing, intelligent manufacturing and knowledge economy, etc [6]. Some scholars believe that intelligent industry refers to the industry that directly uses human's wisdom to conduct research and development, creation, production, management and other activities, and to form tangible or intangible intelligent products to meet social need. Other scholars define the intelligent industry from the realistic perspective, including the following categories in the service industry: telecommunications industries, computer services, software industries, Internet information services, consulting and investigation services, intellectual property services, conference and exhibition services; communication equipment, computer and other electronic equipment manufacturing in the manufacturing industry [7].

Sports industry covers a wide range, through the whole process of sports goods production, sales, stadiums construction activities and sports services, not only enterprises, but also include various participation in industrial activities institutions and self-employed activities, or a use of sports own function and radiation to create value industry, is to provide sports products for the collection of the same kind of economic activities. After the integration of intelligent industry and the concept of sports industry, some scholars put forward that the intelligent sports industry is a multi-integrated industrial chain for the sports industry to realize intelligent information development, and introduce the fine management method of project projects suitable for industrial development. Fine management is a kind of management concept and management technology, which is through the systematic and detailed rules, using procedural, standardized, data and information means, to make the organization management unit accuracy, efficient, collaborative and continuous operation. It can be believed that the practice and development of intelligent sport is actually the process of realizing the fine management concept in each link of the sport industry chain one by one by the help of intelligent technology and information means. After the investigation and research of Wuxi intelligent Sports Industrial Park, relevant scholars described the intelligent sports industry: diversified industrial integration is the direction of the development of "intelligent sport" industry. The sport industry is born with strong industrial integration, and the intelligent sports industry itself is produced by "Internet + sport".

To sum up, the existing concept definition of intelligent sports industry is mostly formed based on the concept of intelligent industry, combined with the characteristics and connotation of intelligent sports and sports industry, and few relevant research documents, the researchers did not agree on the definition of the concept in a strict sense.

\section{References}

1. Feng Maoyan, Jiang Lanzhi. On Smart City and Smart Industry Development — Take Nanjing as an example [J]. Reform and Strategy, 2011,27 (9): 135-136,163.

2. Sun He, Fan Jinling. Where to Start With Developing a Smart Industry [J]. People's Forum, 2017 (06): 94-95

3. National Bureau of Statistics. National Sports Industry Statistics Classification [R]. 2015

4. Ren Zeping. Industrial Trend Observation of China over the next decade [J]. Decision, 2014 (z1): 68-70]

5. Chen Jin, Qiu Kai, Zhang Yuanyuan. Smart Sports Platform Construction Scheme under the background of "Internet" [J]. Journal of Nanjing Institute of Physical Education (Natural Science Edition), 2017, (3): 125-130 
6. Ferretti Luca, Longo Francesco, Merlino Giovanni, Colajanni Michele, Puliafito Antonio, Tapas Nachiket. Verifiable and auditable authorizations for smart industries and industrial Internet-of-Things[J]. Journal of Information Security and Applications, 2021, 59.

7. Bradley David. The building blocks of smart industry[J]. Materials Today,2020,41. 